\title{
Multifunctional to multistage delivery systems: The evolution of nanoparticles for biomedical applications
}

\author{
MARTINEZ Jonathan $\mathrm{O}^{1,2 \dagger}$, BROWN Brandon $\mathrm{S}^{1,2 \dagger}$, QUATTROCCHI Nicoletta ${ }^{1}$, \\ EVANGELOPOULOS Michael $^{1}$, FERRARI Mauro ${ }^{1} \&$ TASCIOTTI Ennio $^{1 *}$ \\ ${ }^{1}$ Department of Nanomedicine, The Methodist Hospital Research Institute, Houston, TX 77006, USA; \\ ${ }^{2}$ Graduate School of Biomedical Sciences, The University of Texas at Houston, Houston, TX 77006, USA
}

Received April 23, 2012; accepted May 29, 2012; published online August 18, 2012

\begin{abstract}
Nanomaterials are advancing in several directions with significant progress being achieved with respect to their synthesis, functionalization and biomedical application. In this review, we will describe several classes of prototypical nanocarriers, such as liposomes, silicon particles, and gold nanoshells, in terms of their individual function as well as their synergistic use. Active and passive targeting, photothermal ablation, and drug controlled release constitute some of the crucial functions identified to achieve a medical purpose. Current limitations in targeting, slow clearance, and systemic as well as local toxicity are addressed in reference to the recent studies that attempted to comprehend and solve these issues. The demand for a more sophisticated understanding of the impact of nanomaterials on the body and of their potential immune response underlies this discussion. Combined components are then discussed in the setting of multifunctional nanocarriers, a class of drug delivery systems we envisioned, proposed, and evolved in the last 5 years. In particular, our third generation of nanocarriers, the multistage vectors, usher in the new field of nanomedicine by combining several components onto multifunctional nanocarriers characterized by emerging properties and able to achieve synergistic effects.
\end{abstract}

nanoparticle, multifunctional, multistage, third generation nanocarriers, porous silicon, cancer, nanomedicine, drug delivery

Citation: Martinez J O, Brown B S, Quattrocchi N, et al. Multifunctional to multistage delivery systems: The evolution of nanoparticles for biomedical applications. Chin Sci Bull, 2012, 57: 3961-3971, doi: 10. 1007/s11434-012-5387-5

Nanotechnology has generated significant enthusiasm in the research setting due to the superior properties materials gain when manufactured at the nanoscale. Current pharmaceuticals fail to operate optimally due to physical and chemicals limitations in areas such as delivery, biodistribution and pharmacokinetics. Nano-based pharmaceuticals, or "nanomedicines", are engineered to either function as a drug or carry a drug while addressing these scientific challenges due to their nano-size. The nanoscale refers to the relative metric size, with one nanometer being equal to one billionth of a meter (roughly the diameter of one molecule of glucose).

$\dagger$ These authors contributed equally to this work.

*Corresponding author (email: etasciotti@tmhs.org)
Nanoparticles (NP) are synthesized from a vast number of raw materials commonly seen on the macroscale, such as gold, lipids, carbon, silica, silicon, and iron [1]. NP made from these raw materials exhibited unprecedented properties not previously seen at the macroscale. Upon this discovery, investigators began exploiting these emerging properties for a variety of applications. The tasks performed by these nanoparticles vary from simple drug protection, as seen in siRNA carriers, to magnetic NP that heat up in the presence of alternating magnetic fields [2]. This review is an attempt to summarize the development of multifunctional nanocarriers from their constitutive parts, discuss some of the existing nanocarriers, and outline the major limitations in the production of these advanced technologies. 


\section{Functional nanocomponents}

Multifunctional nanocarriers can be defined as nanoscale particles capable of performing at least an additional function to that of carrying a therapeutic or imaging payload. There are several prototypical NP worth describing, as they are often the building blocks for larger multifunctional systems [1]. Amongst organic NP, lipid-based NP such as liposomes and micelles, to date, have achieved the most significant clinical success of all NP. Liposomes are 50-300 nm hollow spheres that mimic the composition of the cellular bilayer. They are capable of carrying hydrophilic payloads in their interior, as well as trapping hydrophobic drugs within their lipid membranes. On the contrary, micelles consist of a 10-100 nm spherical monolayer of lipids effectively creating a hydrophobic interior; this allows for the transport of hydrophobic drugs within the micelle's core. Multiple chemotherapeutics have been incorporated in these lipid based NP, some reaching clinical use in humans. The cytotoxic drug doxorubicin has been carried in different NP to represent some of the first clinically used nanomedicines. Doxil, used for breast cancer treatment in the USA, is liposomally encapsulated doxorubicin, and SP1049C, which is currently in phase III clinical trials, is doxorubicin loaded into micelles $[3,4]$. Furthermore, both NP have surfaces that can be modified with polymers or targeting molecules to further enhance their functionality.

Inorganic NP offer functions associated with their electromagnetic or photodynamic properties. For example, hollow gold NP can absorb specific wavelengths of light. In particular, they can be engineered to absorb light within the near infrared (NIR) spectrum. NIR light is known to penetrate deeper into biological tissue without causing ionizing damage. NIR-absorbing hollow gold NP that have been targeted to or injected into a tumor will heat up and destroy tumor cells if an NIR laser is applied to the tumor in a process known as "photothermal ablation" [5]. NP composed of gadolinium are used aspowerful contrast agents providing signals thousands of times greater than background tissue for magnetic resonance imaging (MRI) [6]. Silicon and silica particles have spurred interest in the nanotechnology field due to their versatile and flexible fabrication protocols capable of producing consistent NP with well-controlled sizes and shapes. Both materials can be porosified at the nanoscale allowing the loading of drugs, and their surfaces can be reacted with many different chemical moieties. In particular, the surface can be modified with targeting moieties via bioconjugation techniques in order to tailor the interaction with different types of cells and tissues [7]. Additionally, the highly porous matrices of these two materials serve as potential reservoirs for therapeutics and contrast agents to effectively operate as a carrier for these substances $[8,9]$. The most typical functions associated with NP in the biomedical arena are listed in Table 1. This is not to be considered a complete survey, as an exhaustive discussion of all NP would be out of the scope of this review and has been previously published [1,10-13]. The main limitations of NP such as the lack of selective targeting, bioavailability, and toxicity have fostered the development of delivery systems based on multifunctional NP. To overcome these obstacles, the physicochemical properties of NP such as size, shape,

Table 1 Summary of nanoparticle types being developed for medical applications ${ }^{\text {a) }}$

\begin{tabular}{|c|c|c|c|c|}
\hline Nanoparticle function & Subfunctions & Types of nanoparticles & General medical application & References \\
\hline Drug delivery & $\begin{array}{l}\text { Delayed release } \\
\text { Drug solubilization }\end{array}$ & $\begin{array}{l}\text { Porous silica } \\
\text { Porous silicon } \\
\text { Liposomes } \\
\text { Micelles } \\
\text { Polymer-based NP }\end{array}$ & Deliver drugs to toxic or pathologic cells & {$[14,15]$} \\
\hline Specific targeting & $\begin{array}{l}\text { Immunotargeting } \\
\text { Receptor mediated } \\
\text { Passive targeting }\end{array}$ & $\begin{array}{l}\text { Porous silica } \\
\text { Porous silicon } \\
\text { Liposomes } \\
\text { Micelles } \\
\text { Polymer-based NP }\end{array}$ & $\begin{array}{l}\text { Translocate particles within the body to a } \\
\text { specific site }\end{array}$ & {$[14,16]$} \\
\hline Triggered release & $\begin{array}{l}\mathrm{pH} \text { responsive } \\
\text { Thermoresponsive } \\
\text { Electroresponsive } \\
\text { Ultrasound induced release }\end{array}$ & $\begin{array}{l}\text { Liposomes } \\
\text { Polymer-based NP } \\
\text { Hydrogels }\end{array}$ & $\begin{array}{l}\text { Control the release of a drug or action of } \\
\text { a nanoparticle in a spatiotemporal manner }\end{array}$ & {$[17,18]$} \\
\hline Thermal ablation & Photothermal ablation & $\begin{array}{l}\text { Gold nanoshells } \\
\text { Super-paramagnetic iron } \\
\text { oxide NP }\end{array}$ & Heat up cells or tissue near NP sites & {$[19,20]$} \\
\hline Contrast agents & $\begin{array}{l}\text { MRI agents } \\
\text { X-ray enhancers } \\
\text { Ultrasound }\end{array}$ & $\begin{array}{l}\text { Gadolinium NP } \\
\text { Super-paramagnetic iron } \\
\text { oxide NP }\end{array}$ & Enhance radiological studies & {$[21,22]$} \\
\hline Magnetism & $\begin{array}{l}\text { Localized heating } \\
\text { Particle accumulation }\end{array}$ & $\begin{array}{l}\text { Super-paramagnetic iron } \\
\text { oxide NP }\end{array}$ & $\begin{array}{l}\text { Control particles via magnetic fields } \\
\text { outside the body }\end{array}$ & {$[23,24]$} \\
\hline
\end{tabular}

a) General nanoparticle types are categorized by function and subfunction to deseribe their application towards areas of applied nanomedicine. 
composition and surface properties are currently being investigated in greater detail.

\section{Obstacles in nanomedicine}

Due to the many obstacles the body provides, interest has shifted to engineer a delivery system with multifunctional aspects. In the past, some receptors were originally thought to provide specific targeting due to their high expression on cancer cells [25]; however, when these receptors were conjugated with NP, they exhibited unfavorable side effects. Furthermore, it is important to note that depending on the route of administration, NP will come into contact with various bio-barriers. The physicochemical properties (e.g. size, shape, and chemical composition) of functionalized NP determine the success in overcoming these barriers [26, 27] - further success can be determined based on the NP ability to protect the cargo from degradation. The next possible issue may be in the toxicity of the NP itself once it reaches the target site and releases its payload. Ideally, the NP should be degraded or secreted without producing any adverse effects. Unfortunately, this is not always the case and while no clinically relevant toxicity has been reported, it is too soon to classify NP as completely inert [28-30].

\section{Advancing the hierarchy of nanocarriers}

The aforementioned difficulties encountered with NP, coupled with their limited therapeutic success in the clinic, prompted the nanotechnology community to develop novel classes of NP. Previously, we have proposed that the organization of investigated nanocarriers, or nanovectors, can be effectively categorized into three generations or sub-categories based on their purpose and intended function [31-34] (Figure 1). First generation carriers are composed of the base NP formulation (e.g. liposomes, micelles, gold, etc.) encompassing the agent (e.g. chemotherapeutics, siRNA, DNA, etc.). These first generation nanocarriers were the first NP developed and were approved for clinical use more than 15 years ago (e.g. liposomes). As described earlier, they passively accumulate at target lesions due to microenvironment abnormalities (e.g. fenestrations in the vessel walls of tumors). Second-generation nanocarriers advanced the field by incorporating new functions capable of either shielding or targeting first generation nanocarriers to specific markers found to be overexpressed in the target of choice. These second generation carriers accomplished this by attaching a variety of bio-recognition modalities and demonstrated efficient targeting of tissue abnormalities in vitro, as discussed earlier. Although these carriers have shown promise in animal experiments, they have struggled to achieve clinical efficacy and none to date have achieved approval from the US Food and Drug Administration. Recently, novel classes of nanocarriers have emerged that advance the field by embedding unconventional sophistication into their development. These third generation nanocarriers (TGN) provide a paradigm shift in conventional thought and decouple the multiplicity of tasks required for nanocarriers to navigate intravascular delivery. They provide an elegant and sequential solution to address the common biological barriers (e.g. MPS, endothelial, etc.) encountered resulting in a significant improvement in tumor accumulation. Although they are few in number, TGN are currently under investigation for their immense potential to drastically improve the clinical efficacy while maintaining an overall low therapeutic index, a hallmark of the currently available NP therapeutics. The remainder of this review will be dedicated to highlighting these TGN (Table 2), with a major influence placed on cancer therapy (i.e. treatment and imaging) and introducing our lab's interpretation and solution, the Multistage Silicon Nanocarriers.

\section{Third generation nanocarriers}

The nanotechnology community is constantly redefining nanocarriers aimed at executing several functions to obtain their desired objectives. TGN have been primarily developed
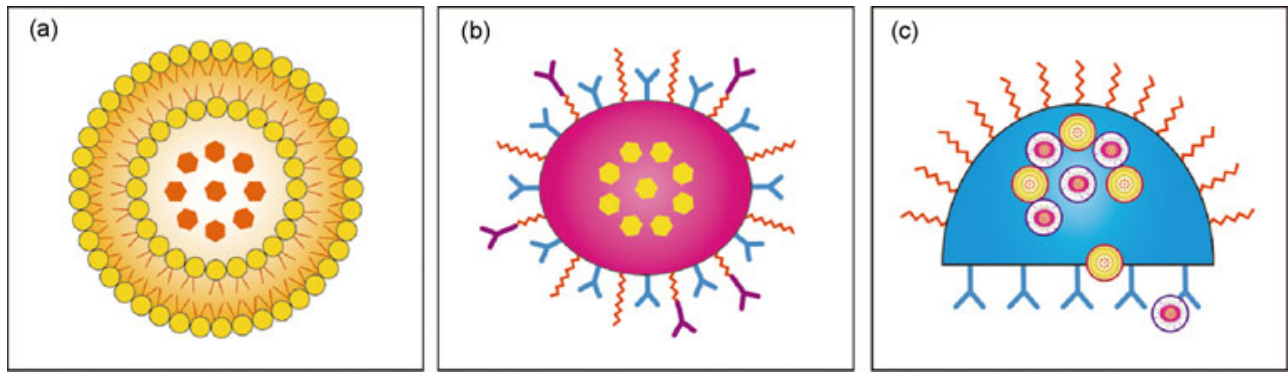

Figure 1 Hierarchy of nanocarriers. (a) First-generation nanocarriers' (e.g. liposomes, micelles) primary role is to enclose therapeutic or diagnostic agents and then localize in tumors by the EPR effect. (b) Second-generation nanocarriers improved by incorporating further modifications allowing for specific targeting via antibodies or other recognition biomolecules or "stealthing" from MPS sequestration. (c) Third-generation nanocarriers advanced the field by creating platforms capable of incorporating and preforming multiple complex functions due to their nanoscale features (e.g. multistage silicon nanocarriers ability to deploy multiple waves of nanoparticles). Reproduced with permission, (C) Wiley-VCH Verlay GmbH \& Co. KGA [33]. 
Table 2 Comparison of first, second, and third generation vectors ${ }^{\mathrm{a})}$

\begin{tabular}{|c|c|c|c|c|}
\hline Generation & Nanocarrier & Size $(\mathrm{nm})$ & Obstacles & Advantages \\
\hline First & $\begin{array}{l}\text { Liposome } \\
\text { Micelles } \\
\text { Gold }\end{array}$ & $\begin{array}{c}50-200 \\
10-100 \\
2-200\end{array}$ & $\begin{array}{l}\text { Deliver a payload to } \\
\text { target lesions }\end{array}$ & $\begin{array}{l}\text { Passively circulate and accumulate due to biological } \\
\text { abnormalities }\end{array}$ \\
\hline Second & $\begin{array}{l}\text { Bio-recognition modalities } \\
\text { attached to first generation } \\
\text { nanocarriers }\end{array}$ & & $\begin{array}{l}\text { Inability to accumu- } \\
\text { late at target tissue }\end{array}$ & $\begin{array}{l}\text { Attaching bio-recognition modalities allows for } \\
\text { exploitation of target tissue abnormalities }\end{array}$ \\
\hline \multirow{5}{*}{ Third } & $\begin{array}{l}\text { Multistage silicon } \\
\text { nanocarriers }\end{array}$ & $\begin{array}{l}\text { Diameter } \\
500-3000 \mathrm{~nm} \\
\text { Pores } \\
3-150 \mathrm{~nm}\end{array}$ & $\begin{array}{l}\text { Biological barriers } \\
\text { impede successful } \\
\text { delivery of payload }\end{array}$ & $\begin{array}{l}\text { Navigate blood stream } \\
\text { Recognize diseased vasculature } \\
\text { Retain and release payload } \\
\text { Ability to elicit a signaling or biological cascade, } \\
\text { resulting in receptors able to attract agents }\end{array}$ \\
\hline & Nanoshells & $100-200 \mathrm{~nm}$ & & $\begin{array}{l}\text { Absorb light in NIR allowing for external activation } \\
\text { Absorb or scatter light enabling several imaging } \\
\text { applications }\end{array}$ \\
\hline & Nanorods & $\begin{array}{l}\text { Length } \\
15-70 \mathrm{~nm} \\
\text { Aspect ratio } \\
1.7-4.4 \mathrm{~nm}\end{array}$ & & $\begin{array}{l}\text { In vivo communication/signaling yielding } \mathrm{NP} \\
\text { amplification }\end{array}$ \\
\hline & Nanocell & $180-200 \mathrm{~nm}$ & & Temporal release of payload \\
\hline & Protocell & $100-150 \mathrm{~nm}$ & & Stability to lipid membrane and increase in delivery agent \\
\hline
\end{tabular}

a) Different generations were compared using size, intended obstacles they could accomplish, and advantages.

to tackle the heterogeneous nature of tumors, but can potentially be applied to several disorders based on the versatility of the systems. TGN contain a complex nano-sized feature that imparts an innovative trait allowing for an unprecedented function not available in second-generation carriers. Examples of these carriers include gold-containing NP, which can use tunable optical properties to absorb light in the NIR, thus allowing for external activation in the clinic due to the low absorption of biologics (i.e. water and hemoglobin) within this region [35] and integrate other properties based on gold's versatility. Solid gold NP display surface plasmon resonance throughout the visible spectrum red shifting with increases in size (spherical) or aspect ratio (rods) [36]. However NIR absorption is not the case for all gold NP, for example, spherical gold NP display absorption spectra with minor redshifts as the diameter increases, with peak absorbance ranging from 520 to $600 \mathrm{~nm} \mathrm{[36].} \mathrm{On} \mathrm{the}$ other hand, gold nanorod's (Figure 2(a)) resonance fluctuates based on their aspect ratio (length/width), and with increases to this ratio, their spectra significantly shifts well into the NIR region, with peak absorbance ranging from 600 to $900 \mathrm{~nm}$ [36]. Another gold-containing NP example is nanoshells (Figure 2(a)). Nanoshells typically range from 100 to $200 \mathrm{~nm}$ in diameter, and are composed of a dielectric core enclosed by a thin metallic (typically gold) layer with tunable properties [37,38]. The archetypical nanoshell involves a silica core (110-120 nm diameter) with a $10 \mathrm{~nm}$ layer of gold resulting in peak surface plasmon resonance at $800 \mathrm{~nm}$, capable of providing photothermal therapy using NIR excitation [39-41]. As with gold spheres and rods, a similar phenomenon was discovered with gold nanoshells where decreases in the thickness of the outer gold layer would significantly redshift the absorption spectra well into the NIR region [42]. Moreover, one can vary the core/shell ratio of nanoshells to affect their ability to absorb or scatter light, thus enabling their use not only for photothermal applications but also for several imaging applications [41-43]. In addition, fluorescent enhancement of NIR dyes without alteration of the photothermal properties can be achieved by using a silica spacer on nanoshells $[44,45]$ and with human serum albumin for shells and rods [46] thus enabling significant increases in fluorescent intensity due to nanoscale effects. Furthermore, dual magnetic resonance and optical imaging enhancement was also achieved using a silica layer by doping it with iron oxide and NIR dye on a nanoshell, resulting in a platform capable of targeting (via monoclonal antibody), photothermal activation, and imaging [47]. Recently, the labs of Zhang and Li [5,48-51] have demonstrated that hollow gold nanospheres retain the photothermal properties of nanoshells and have used them for cancer applications and have incorporated them with chemotherapeutic agents such as doxorubicin. Another gold-related approach incorporates the intrinsic properties of spherical gold NP and bacteriophages to create self-assembled networks of gold NP-embedded phages (Au-phage) with unique properties that emerge from this synergism [52]. These Au-phages (Figure 2(b)) spontaneously organize and maintain the biological properties of phages, including their ability to target cells, coupled with the unique properties of gold NP. Moreover, they can act as enhanced and superior reporters for fluorescence and dark field microscopy and provide a redshift in gold NP absorbance, allowing for NIR absorption for in vivo photothermal therapy [52,53]. Gold nanoshells, rods, and hollow spheresare considered TGN based on their nano-sized features that enable novel properties to emerge that will facilitate success in the clinic attributed to their ability to modulate NIR absorbance. Spherical gold NP alone are not considered TGN due to their ability to only absorb light shorter than $600 \mathrm{~nm}$, however when integrated with phage technology novel properties 

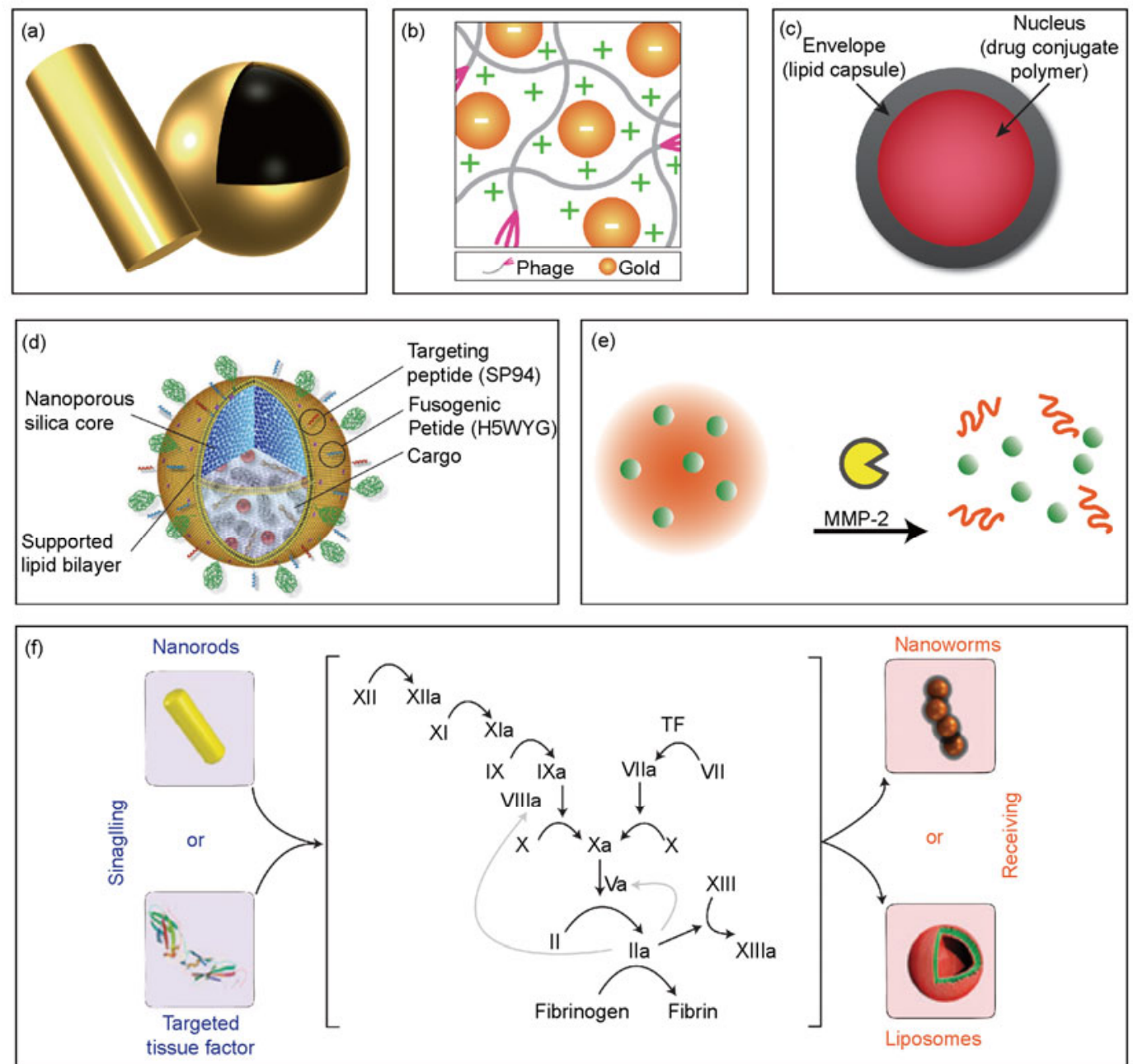

Figure 2 Third-generation nanocarrier platforms. (a) Gold nanoshells and rods display unique photothermal properties and can enhance fluorescent and magnetic properties when coupled to their surface. (b) Au-phage networks are the result of the unique synergism yielded upon the incorporation of gold nanoparticles with bacteriophages, schematic is shown here displaying the effect of charge. Adapted from [52] and reproduced by permission from Jonathan O. Martinez, Methods in Bioengineering: Nanoscale Bioengineering and Nanomedicine, Norwood, MA: Artech House, Inc., 2009. (C) 2009 by Artech House, Inc. (c) Nanocells are comprised of two compartments (nucleus and envelope) which allows for the temporal release of agents enabling time-dependent delivery of therapeutics, adapted from [54]. (d) Protocells are nanoporous silica cores supported by a lipid bilayer. The porous core allows for the incorporation of a diverse array of cargoes, while the lipid bilayer enables for further conjugation of targeting and fusogenic peptides yielding an agent capable of providing a "one nanoparticle, one kill". Reproduced with permission from NPG [55]. (e) Embedded nanoparticles enable the concentrated delivery of smaller nanoparticles deep within the tumor site by taking advantage of the responsive nature of larger nanoparticles to tumor microenvironment cues (e.g. Gelatin \& MMP-2). Reproduced from [60] with permission from National Academy of Sciences, USA. (f) Communicating nanoparticles benefit from the amplification of signals created through biological cascades resulting in significant increases in nanoparticle tumor accumulation. Using the increases in factors involved in the coagulation cascade, via the heating of gold nanorods, one could effectively increase the accumulation of targeted therapeutic and diagnostic agents. Reproduced with permission from NPG [61].

emerge including the ability to home to targets and enhanced NIR heating all in a single, self-assembled package. Furthermore, these NP can be decorated with biorecognition moieties or polymers as with second generation carriers.

TGN could also be synthesized or fabricated to have cell-mimicking coatings or properties. These inherited properties allow these nanocarriers an advanced degree of integration into the hosts system by mimicking cells commonly encountered during targeting. One such system was devised to co-deliver two anti-cancer drugs imparting distinct release profiles to each drug [54]. This system was composed of a central polymer-based NP containing drug A encircled by a PEGylated-lipid compartment, which captured and retained drug B until the outer lipid layer was completely compromised. These "nanocells" (Figure 2(c)) allowed for the temporal release of the entrapped drugs, and the proof-of-principle was demonstrated by trapping the anti-angiogenesis agent combretastatin in the envelope/lipid capsule of the nanocell. A doxorubicin-PLGA conjugate was used to form the nucleus and concentrate doxorubicin within the core of nanocells. Sengupta et al. [54] created $180-200 \mathrm{~nm}$ carriers and illustrated that by using this novel approach a synergistic combination could be achieved. Spe- 
cifically, the anti-angiogenesis agent lead to the collapse of tumor associated vessels resulting in the effective entrapment of nuclear NP within the tumor where they could provide a steady release of doxorubicin, killing the remainder of the cells. A similar, yet different approach uses a nanoporous silica NP nucleus supported by a lipid bilayer membrane (i.e. liposomes) resulting in a carrier of 100-150 $\mathrm{nm}$. Moreover, the lipid bilayer membrane can incorporate further functionalization (for targeting, endosomal disruption, and shielding) while the silica core absorbs and retains multiple combinations of agents allowing for potential drug cocktails. Ashley et al. [55] demonstrated that the silica core of these "protocells" (Figure 2(d)) imparted significant stability to the lipid membranes, displaying a 100-fold increase in targeting cells, and 1000-fold increase in the delivery of agents (chemotherapeutics, siRNA, QD) when compared with clinically prepared liposomes [56]. Furthermore, protocells impressively demonstrated an unprecedented 10000fold increased affinity for human hepatocellular carcinoma. This enhancement was so potent that when carrying a cocktail of chemotherapeutics, a single protocell was capable of killing a single cancerous cell. An alternative approach is to fabricate carriers that mimic red blood cells (RBC) [57-59], a major constituent of blood. Doshi et al. [57] synthesized RBC-like particles by using a PLGA template, followed by layer-by-layer addition and protein cross-linking yielding carriers capable of carrying oxygen, flowing through capillaries, and encapsulate a variety of agents, including NP. In a related study, Merkel et al. [58] observed that carriers designed to match the elastic modulus of RBC were found to extend their circulation time in blood. On the other hand, rather than synthesizing carriers of similar shape and size, $\mathrm{Hu}$ et al. [59] imparted RBC-like properties by camouflaging the surface of PLGA NP with natural erythrocyte membranes. This RBC-coated NP demonstrated superior blood circulation and maintained the associated proteins of natural RBC. The biomimetic strategies discussed provide powerful solutions to conventional second generation carriers by enabling enhanced targeting, co-delivery of drug cocktails, and improved blood circulation.

Other TGN function by relying on the synergism and cooperation of multiple NP resulting in superior enhancement in delivery and therapy of agents $[60,61]$. One such example of NP synergism was proposed by developing a system where a tumor "sensing" (i.e. reactive to cues found within tumor microenvironment) NP would contain several smaller NP within its core (Figure 2(e)). The rationale behind this approach was to retain the properties of $100 \mathrm{~nm}$ NP (e.g. passive targeting, long circulation) and upon reaching the tumor, the larger NP would degrade forcing the release of smaller NP to deeply penetrate the dense matrix of tumors typically hindered by larger NP. Wong et al. [60] demonstrated a proof-of-principle using $100 \mathrm{~nm}$ gelatin NP containing $10 \mathrm{~nm}$ QD which when triggered by proteases, (e.g. matrix metalloproteinases) would cause degradation or shrinkage of the larger gelatin particles allowing the QD to diffuse deeper into tumor tissue. This NP system conserved the long circulation time and tumor penetration (via EPR effect) assets pertaining to 100-200 nm NP while also coupling the favorable attributes of smaller NP to deliver agents with significantly improved tumor penetration. Yet another strategy relies on using NP to elicit a signaling or biological cascade, which in turn creates an abundance of receptors that can attract agents (Figure 2(f)). von Maltzahn et al. [61] proved this strategy by taking advantage of the coagulation cascade and constructed systems to intensify disease targeting. The designs of these systems relied on the synergism of "signaling" (NP or engineered proteins) and "receiving" (therapeutic or diagnostic) modules to activate the coagulation cascade, thus relaying the location and attracting the circulating clot-targeted NP. Signaling components explored here include gold nanorods and RGD-targeted human tissue factor which elicited the activation of the coagulation cascade by photothermal disruption of tumor vessels and by binding to $\alpha_{\mathrm{v}} \beta_{3}$ in tumor blood vessels, respectfully. This "signaling" activation enabled communication to recruit and amplify receiving components to the site of the signaling carriers. The investigators confirmed the amplified recruitment of diagnostic (iron oxide nanoworms) and therapeutic (doxorubicin-loaded liposomes) receiving components through the tethering of active coagulation transglutaminase FXIII or targeted fibrin. This in vivo communication resulted in autonomous operation, increasing the targeting 40-fold over non-communicating NP. The discussed strategies established novel methods in the delivery and recruitment of NP to diseased sites, specifically to tumors, and provided simple methods to increase the delivery while reducing the potent toxicity of chemotherapeutics.

\section{Multistage silicon nanocarriers}

Our laboratory envisioned, designed, and engineered Multistage Silicon Nanocarriers (MSN), a unique and innovative example of TGN. Our strategy was to decouple the multiplicity of tasks required to a single NP and distribute them onto multiple stages. According to this approach, the first stage carrier is responsible for the navigation through the bloodstream, the avoidance of MPS, the recognition of the diseased vasculature, and the retention and release of the embedded second-stage particles $[62,63]$. The second-stage particles are NP able to extravasate through vessels' fenestrations and diffuse in the tumor parenchyma, thus concentrating diagnostic and therapeutic agents within the target microenvironment. In addition, these second stage NP can be functionalized with cancer specific ligands allowing for cell-specific targeting within the tumor (Figure 3(a)-(c)).

Obtaining clinical success requires carriers to be designed such that they meet a stringent set of criteria. A critical aspect of the MSN design is the appropriate material for 

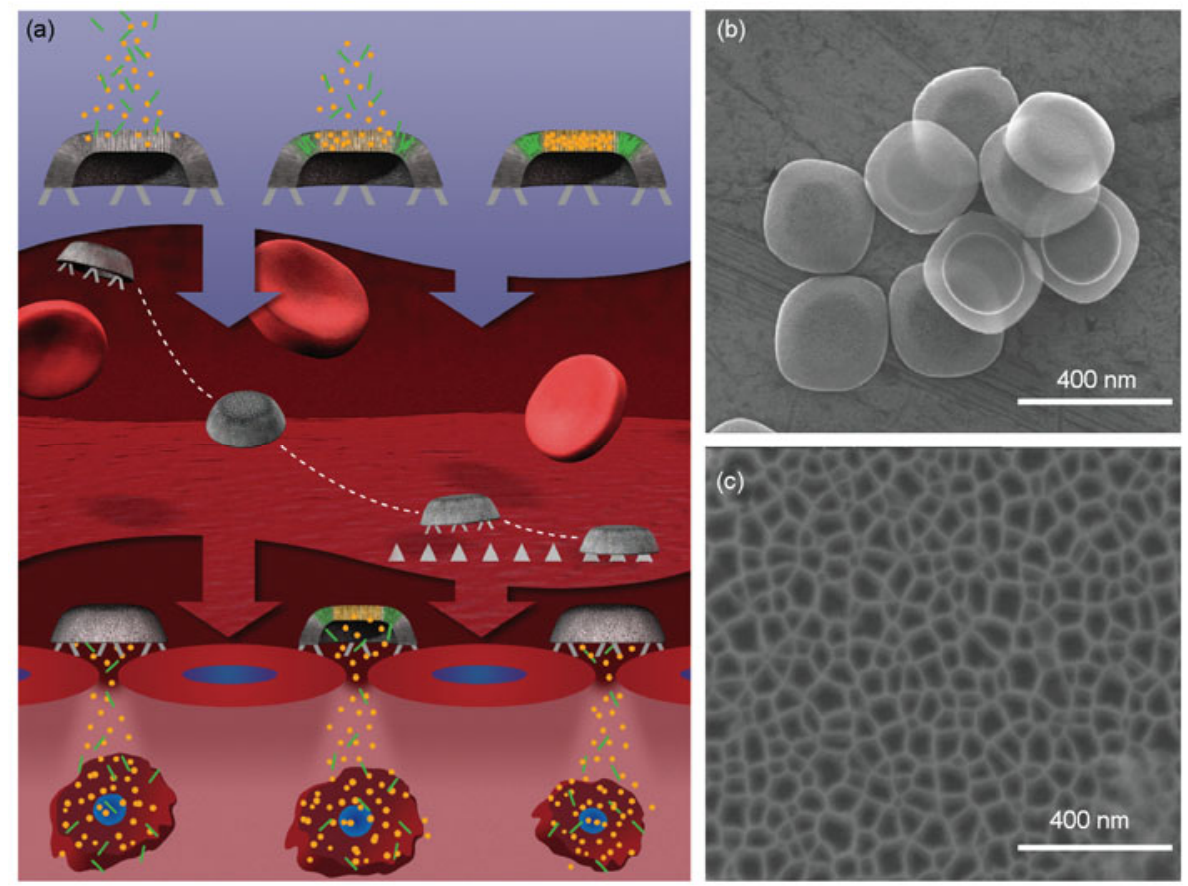

Figure 3 Schematic and SEM micrographs describing the process and shape of multistage silicon nanocarriers. (a) MSN is initially loaded with second-stage NP into the pores of the silicon nanocarriers. Upon systemic administration, the rational design of MSN allows them to travel within the bloodstream, avoid MPS sequestration, and preferentially drift towards the target's endothelium and firmly adhere. Once docked, MSN can release their cargo (i.e. second stage NP) that will infiltrate into the target's microenvironment where they can specifically target diseased cells and fulfill their final objective. (b) Overall view of a view MSN illustrating the uniform size and shape within each fabrication batch. (c) Magnified image of the nano-sized pores of MSN, which can serve to accommodate a variety of NP. Reproduced with permission from [62].

the first stage. This material must be biocompatible, biodegradable, versatile surface for chemical modification, and flexible fabrication schemes enabling defined control over shape and size. With this in mind, we chose porous silicon as the material of choice [64-67], whose porosity would be able to accommodate a broad range of NP. By using advanced modeling of the blood rheology, margination (i.e. the propensity to drift towards vessel walls) and flow dynamics, vascular targeting, vessel permeability, and endothelial adhesion and internalization, we rationally designed MSN to contain the optimal attributes for optimal intravascular delivery [68-74]. We demonstrated the ability to fabricate carriers in several shapes (e.g. hemispherical, discoidal, and cylindrical), sizes (400-3000 nm) and pore sizes (3-150 nm) yielding monodispersed particles [9] (Figure 3(d),(e)) capable of retaining a variety of NP (Figure 4) (e.g. QD [62], carbon nanotubes [62,75], fullerenes [75], nanoliposomes [76], iron-oxide [77,78], and gold [79,80]). Furthermore, the in vitro and in vivo degradation of MSN was verified and demonstrated no significant adverse reactions [81]. Additionally, MSN were found to degrade in various physiological environments (PBS, serum, media, and blood) into orthosilicic acid and their degradation rate could be tailored through PEGylation [82,83]. Investigating the in vitro interactions of MSN with endothelial and macrophage cells illustrated no effect on their proliferation, cell cycle, or apoptosis, and failed to produce an increase in the expression of several inflammatory cytokines [79,82,83]. Similar results were revealed in vivo when investigating the effect of acute and sub-chronic administration of MSN, which demonstrated within the span of a month no significant release of biochemical markers or cytokines in the blood plasma of exposed mice [84]. Furthermore, the ability of MSN to marginate and localize to the diseased endothelium is highly dependent on its shape and size, and has been extensively studied within our group [69-71]. We concluded that when used for intravascular delivery, discoidal-shaped MSN yielded the highest concentration of silicon in tumors, all the while reducing accumulation in the liver [85]. Expanding on these results, our group examined the effect of size on the distribution of discoidal (or "plateloid") MSN by coupling intravital microscopy with elemental analysis. van de Ven et al. [86] determined that 1000 nm MSN preferentially accumulated in tumors at higher numbers and concentration than 600 and $1800 \mathrm{~nm}$ carriers. Furthermore, our group reported an unprecedented concentration of MSN to rapidly (less than $1 \mathrm{~h}$ ) emerge at tumor sites at almost $10 \%$ of the injected dose per gram of organ for RGD-targeted carriers. In addition, Mann et al. [87] displayed enhanced vascular targeting of MSN to the bone marrow using an E-selectin thioaptamer covalently linked to its surface and retained the ability to load NP.

The loading and release of NP relies on several conditions, as well as synergism between the dimensions, charge 

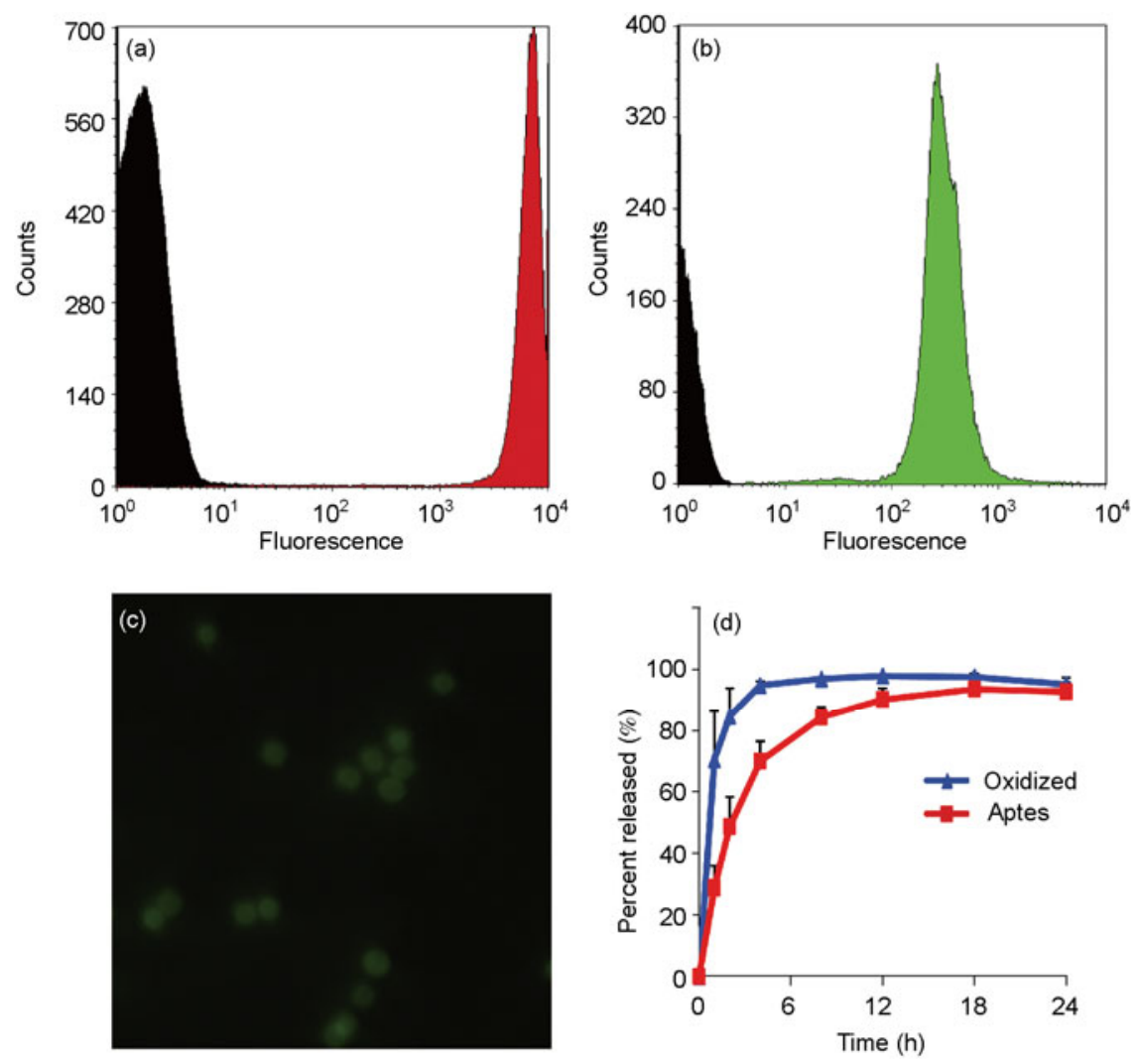

Figure 4 Loading and release of nanoparticles from MSN. (a)-(c) Loading of NP (liposomes, quantum dots) into MSN. (a), (b) Flow cytometry of loading comparing the fluorescence of MSN, either unloaded (black) or with NP-loaded ((a) green, liposomes; (c) red, quantum dots). (c) Fluorescent image of MSN containing FITC labeled nanoliposomes. (d) Release profiles of quantum dots from oxidized (blue) and APTES modified (red) MSN.

and concentration of the payload and MSN [31,62]. Using a $1000 \mathrm{~nm}$ MSN as an example, we calculated that each MSN would be able to accommodate $3.8 \times 10^{5} \mathrm{NP}$ (assuming a 10 $\mathrm{nm}$ diameter). Experimental results, using $10 \mathrm{~nm}$ iron oxide $\mathrm{NP}$, revealed that we could effectively load $0.3 \mathrm{pg}$ of iron $\left(\sim 1 \times 10^{5} \mathrm{NP}\right)$ per MSN [31]. In addition, the surface of MSN represents a suitable platform for covalent and electrostatic attachment of fluorescent dyes, radioactive molecules, proteins, polymers, and other NP $[83,88,89]$. For example, bacteriophage networks (phages + gold NP) have been attached to MSN based on electrostatic interactions producing multifunctional nanoassemblies [31]. We have also demonstrated the controlled and stable release of biomolecules through PLGA/MSN complexes and agarose surface coatings thus enabling intermediate release profiles of the embedded biomolecules (Figure 5) [88,89]. In addition, NP retention and release could be controlled by conjugating silanes to the surface of MSN that imparted a delayed release when compared to uncoated carriers and was capable of retaining the payload in vivo [77].

Furthermore upon loading with NP, MSN exhibit enhanced properties capable of providing novel and greatly improved imaging and therapeutic agents. Ananta et al. [75] illustrated that by loading MSN with gadolinium-based contrast agents, incorporated within carbon NP, would dra- matically alter the longitudinal relaxivity for MRI resulting in an 40-fold increase over clinically used agents. Moreover, loading MSN with iron oxide NP resulted in constructs with shorter relaxation times, providing increased negative contrast for T2 weighted MRI [77]. Enhanced efficacy using MSN was achieved by delivering nanoliposomes containing siRNA to orthotropic models of ovarian cancer. After a single administration of siRNA loaded MSN, prolonged gene silencing and decreased tumor burden was observed over three weeks [81]. On the other hand, to achieve a similar effect with nanoliposomes alone required multiple doses a week. The proposed mechanism for this sustained delivery relies on the slow degradation of MSN in vivo, and thus this approach could be applicable for the long-term release of several embedded NP resulting in less doses needed to treat tumors. Recently, Shen et al. [80] discovered that upon loading MSN with NIR responsive NP (hollow gold NP), a significant increase in heat generation and killing of breast tumor cells (regardless of genetic background) was observed. This cooperative thermal therapy packaging between MSN and hollow gold NP revealed benefits that red shifted the absorption enabling deeper penetration within tissues. Furthermore, the nanoscale organization within MSN permitted more efficient energy-to-heat conversion resulting in a strengthened therapeutic response. Hence, by 

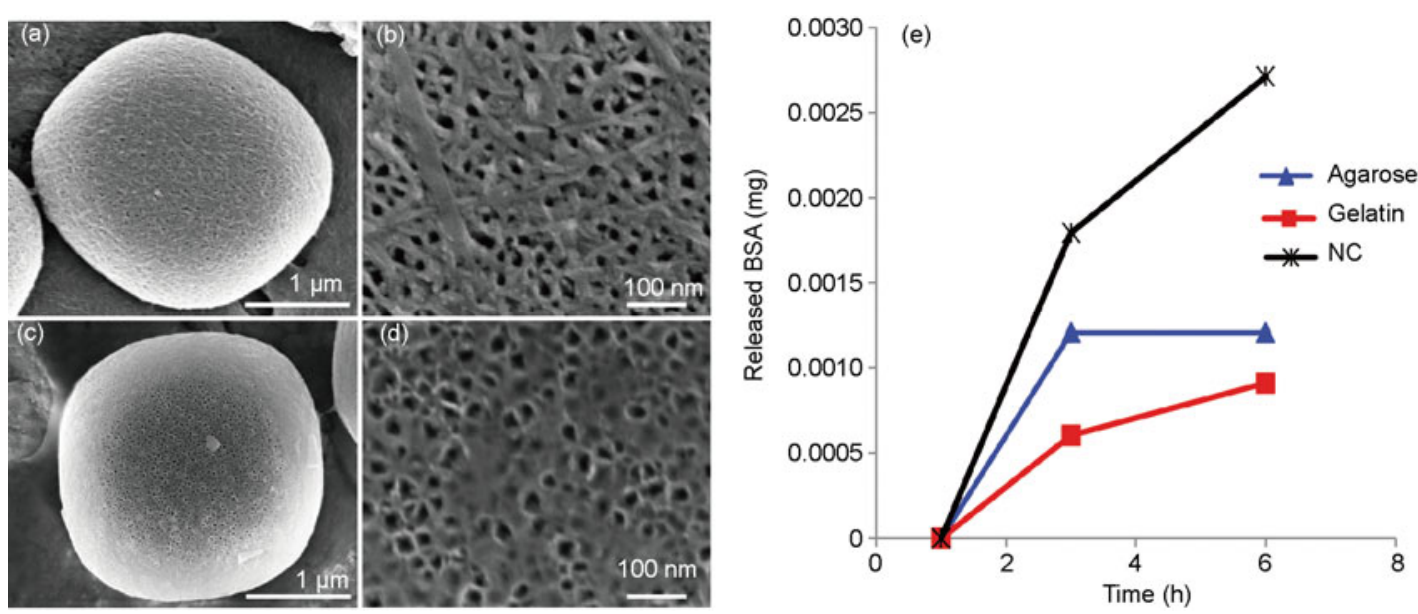

Figure 5 Coating and delayed release of biomolecules from MSN. (a)-(d) SEM micrographs demonstrating the coating of MSN with agarose ((a),(b)) and gelatin ((c),(d)) at the single particle scale ((a),(c)) and pore scale ((b),(d)). (e) Release profiles of BSA-FITC from uncoated (black), agarose (blue), and gelatin (red) coated MSN. The coating of MSN yielded a delay in the release of BSA enabling kinetics not possible without a coating.

loading NP into MSN novel features emerged resulting in an unprecedented amplification in function.

\section{Conclusion}

In conclusion, multifunctional nanocarriers are the product of cumulative research in the nanotechnology field applied to modern medicines. Third generation nanocarriers represent the latest technology designed to address multiple clinical demands while simultaneously addressing limitations of conventional nanoparticles. Multifunctional nanocarriers represent the products of an ever-growing array of "nano" building blocks; the most relevant to our work corresponds to the Multistage Silicon Nanocarriers. This has been seen with the delivery of both contrast agents and bioactive drugs for the diagnosis and treatment within a contained system. However, issues still remain with regard to the assessment and verification of in vivo cytotoxicity, biocompatibility testing, and clinical approvals. These remain paramount areas of focus in developing next generation nanocarriers that have been designed specifically to address human diseases. Future formulations can be expected to incorporate newly designed nanomaterials that leverage existing constructs such as protocells and multistage particles to enhance the potential attributes of future nano-based delivery platforms.

The authors would like to thank Enrica De Rosa for providing valuable data, Matt Landry for image preparation, Alessandro Parodi, Shilpa Scar$i a$, and Michael Frazier for their assistance on manuscript preparation, and discussion. JOM was supported by Award Number TL1RR024147 from the National Center for Research Resources (NCRR).

1 Sanvicens N, Marco M P. Multifunctional nanoparticles-properties and prospects for their use in human medicine. Trends Biotechnol,
2008, 26: 425-433

2 Babincova M, Altanerova V, Altaner $\mathrm{C}$, et al. In vivo heating of magnetic nanoparticles in alternating magnetic field. Med Phys, 2004, 31: 2219-2221

3 Pastan I, Hassan R, Fitzgerald D J, et al. Immunotoxin therapy of cancer. Nat Rev Cancer, 2006, 6: 559-565

4 O'Brien M E, Wigler N, Inbar M, et al. Reduced cardiotoxicity and comparable efficacy in a phase III trial of pegylated liposomal doxorubicin hcl (caelyx/doxil) versus conventional doxorubicin for first-line treatment of metastatic breast cancer. Ann Oncol, 2004, 15: $440-449$

5 Melancon M P, Lu W, Yang Z, et al. In vitro and in vivo targeting of hollow gold nanoshells directed at epidermal growth factor receptor for photothermal ablation therapy. Mol Cancer Ther, 2008, 7: 17301739

6 Ghaghada K B, Ravoori M, Sabapathy D, et al. New dual mode gadolinium nanoparticle contrast agent for magnetic resonance imaging. PLoS One, 2009, 4: e7628

7 Tang F, Li L, Chen D. Mesoporous silica nanoparticles: Synthesis, biocompatibility and drug delivery. Adv Mater, 2012, 24: 1504-1534

8 Qian K K, Bogner R H. Application of mesoporous silicon dioxide and silicate in oral amorphous drug delivery systems. J Pharm Sci, 2012, 101: 444-463

9 Chiappini C, Tasciotti E, Fakhoury J, et al. Tailored porous silicon microparticles: Fabrication and properties. Chem Phys Chem, 2010, doi: 10.1002/cphc.200900914

10 Longmire M, Choyke P L, Kobayashi H. Clearance properties of nano-sized particles and molecules as imaging agents: Considerations and caveats. Nanomedicine (Lond), 2008, 3: 703-717

11 Musacchio T, Torchilin V P. Recent developments in lipid-based pharmaceutical nanocarriers. Front Biosci: A J Virtual Libr, 2011, 16: $1388-1412$

12 van Vlerken L E, Amiji M M. Multi-functional polymeric nanoparticles for tumour-targeted drug delivery. Expert Opin Drug Deliv, 2006, 3: 205-216

13 Wu S H, Hung Y, Mou C Y. Mesoporous silica nanoparticles as nanocarriers. Chem Commun (Camb), 2011, 47: 9972-9985

14 Du Y Z, Cai L L, Li J, et al. Receptor-mediated gene delivery by folic acid-modified stearic acid-grafted chitosan micelles. Int J Nanomed, 2011, 6: 1559-1568

15 Shin $\mathrm{H} \mathrm{C}$, Alani A W, Cho $\mathrm{H}$, et al. A 3-in-1 polymeric micelle nanocontainer for poorly water-soluble drugs. Mol Pharm, 2011, 8: $1257-1265$

16 Thierry B, Al-Ejeh F, Brown M P, et al. Immunotargeting of functional nanoparticles for MRI detection of apoptotic tumor cells. 
Adv Mater, 2009, 21: 541-545

17 Jing $\mathrm{Y}$, Zhu $\mathrm{Y}$, Yang $\mathrm{X}$, et al. Ultrasound-triggered smart drug release from multifunctional core-shell capsules one-step fabricated by coaxial electrospray method. Langmuir, 2011, 27: 1175-1180

18 Muhammad F, Guo M, Qi W, et al. Ph-triggered controlled drug release from mesoporous silica nanoparticles via intracelluar dissolution of zno nanolids. J Am Chem Soc, 2011, 133: 8778-8781

19 Letfullin R R, Iversen C B, George T F. Modeling nanophotothermal therapy: Kinetics of thermal ablation of healthy and cancerous cell organelles and gold nanoparticles. Nanomedicine, 2011, 7: 137-145

20 Stern J M, Stanfield J, Kabbani W, et al. Selective prostate cancer thermal ablation with laser activated gold nanoshells. J Urol, 2008, 179: 748-753

21 Hedlund A, Ahren M, Gustafsson H, et al. Gd(2)o(3) nanoparticles in hematopoietic cells for MRI contrast enhancement. Int $\mathbf{J}$ Nanomed, 2011, 6: 3233-3240

22 Toth E, Bolskar R D, Borel A, et al. Water-soluble gadofullerenes: Toward high-relaxivity, $\mathrm{pH}$-responsive MRI contrast agents. J Am Chem Soc, 2005, 127: 799-805

23 Hasenpusch G, Geiger J, Wagner K, et al. Magnetized aerosols comprising superparamagnetic iron oxide nanoparticles improve targeted drug and gene delivery to the lung. Pharm Res, 2012, doi: http://dx.doi.org/10.1007/s11095-012-0682-Z

24 Yigit M V, Moore A, Medarova Z. Magnetic nanoparticles for cancer diagnosis and therapy. Pharm Res, 2012, doi: http://dx.doi.org/ 10.1007/s11095-012-0679-7

25 Wang X, Li J, Wang Y, et al. Hft-t, a targeting nanoparticle, enhances specific delivery of paclitaxel to folate receptor-positive tumors. ACS Nano, 2009, 3: 3165-3174

26 Moghimi S M, Hunter A C, Murray J C. Long-circulating and target-specific nanoparticles: Theory to practice. Pharmacol Rev, 2001, 53: 283-318

27 Howard M D, Jay M, Dziubla T D, et al. Pegylation of nanocarrier drug delivery systems: State of the art. J Biomed Nanotechnol, 2008, 4: $133-148$

28 Linkov I, Satterstrom F K, Corey L M. Nanotoxicology and nanomedicine: Making hard decisions. Nanomedicine, 2008, 4: 167171

29 Nel A, Xia T, Madler L, et al. Toxic potential of materials at the nanolevel. Science, 2006, 311: 622-627

30 Suh W H, Suslick K S, Stucky G D, et al. Nanotechnology, nanotoxicology, and neuroscience. Prog Neurobiol, 2009, 87: $133-170$

31 Godin B, Tasciotti E, Liu X, et al. Multistage nanovectors: From concept to novel imaging contrast agents and therapeutics. ACC Chem Res, 2011, 44: 979-989

32 Sakamoto J H, van de Ven A L, Godin B, et al. Enabling individualized therapy through nanotechnology. Pharmacol Res, 2010 , 62: 57-89

33 Godin B, Serda R E, Sakamoto J, et al. Nanoparticles for cancer detection and therapy. Nanotechnology. New York: Wiley-VCH Verlag GmbH \& Co. KGaA, 2010

34 Riehemann K, Schneider S W, Luger T A, et al. Nanomedicinechallenge and perspectives. Angew Chem Int Edi, 2009, 48: 872-897

35 Weissleder R. A clearer vision for in vivo imaging. Nat Biotechnol, 2001, 19: 316-317

36 Huang X, Jain P K, El-Sayed I H, et al. Plasmonic photothermal therapy (PPTT) using gold nanoparticles. Lasers Med Sci, 2008, 23: 217-228

37 West J L, Halas N J. Engineered nanomaterials for biophotonics applications: Improving sensing, imaging, and therapeutics. Annu Rev Biomed Eng, 2003, 5: 285-292

38 Hirsch L R, Gobin A M, Lowery A R, et al. Metal nanoshells. Ann Biomed Eng, 2006, 34: 15-22

39 Hirsch L R, Stafford R J, Bankson J A, et al. Nanoshell-mediated near-infrared thermal therapy of tumors under magnetic resonance guidance. Proc Natl Acad Sci USA, 2003, 100: 13549-13554

40 O'Neal D P, Hirsch L R, Halas N J, et al. Photo-thermal tumor ablation in mice using near infrared-absorbing nanoparticles. Cancer
Lett, 2004, 209: 171-176

41 Gobin A M, Lee M H, Halas N J, et al. Near-infrared resonant nanoshells for combined optical imaging and photothermal cancer therapy. Nano Lett, 2007, 7: 1929-1934

42 Loo C, Lin A, Hirsch L, et al. Nanoshell-enabled photonics-based imaging and therapy of cancer. Technol Cancer Res Treat, 2004, 3 : 33-40

43 Loo C, Lowery A, Halas N, et al. Immunotargeted nanoshells for integrated cancer imaging and therapy. Nano Lett, 2005, 5: 709-711

44 Tam F, Goodrich G P, Johnson B R, et al. Plasmonic enhancement of molecular fluorescence. Nano Lett, 2007, 7: 496-501

45 Bardhan R, Grady N K, Halas N J. Nanoscale control of near-infrared fluorescence enhancement using Au nanoshells. Small, 2008, 4: 1716-1722

46 Bardhan R, Grady N K, Cole J R, et al. Fluorescence enhancement by Au nanostructures: Nanoshells and nanorods. ACS Nano, 2009, 3: 744-752

47 Bardhan R, Chen W X, Perez-Torres C, et al. Nanoshells with targeted simultaneous enhancement of magnetic and optical imaging and photothermal therapeutic response. Adv Funct Mater, 2009, 19: 3901-3909

48 Schwartzberg A M, Olson T Y, Talley C E, et al. Synthesis, characterization, and tunable optical properties of hollow gold nanospheres. J Phys Chem B, 2006, 110: 19935-19944

49 Lu W, Xiong C, Zhang G, et al. Targeted photothermal ablation of murine melanomas with melanocyte-stimulating hormone analogconjugated hollow gold nanospheres. Clin Cancer Res, 2009, 15: 876-886

50 You J, Shao R, Wei X, et al. Near-infrared light triggers release of paclitaxel from biodegradable microspheres: Photothermal effect and enhanced antitumor activity. Small, 2010, 6: 1022-1031

51 You J, Zhang G, Li C. Exceptionally high payload of doxorubicin in hollow gold nanospheres for near-infrared light-triggered drug release. ACS Nano, 2010, 4: 1033-1041

52 Souza G R, Christianson D R, Staquicini F I, et al. Networks of gold nanoparticles and bacteriophage as biological sensors and celltargeting agents. Proc Natl Acad Sci USA, 2006, 103: 1215-1220

53 Souza G R, Staquicini F I, Christianson D R, et al. Combinatorial targeting and nanotechnology applications. Biomed Microdevices, 2010, 12: 597-606

54 Sengupta S, Eavarone D, Capila I, et al. Temporal targeting of tumour cells and neovasculature with a nanoscale delivery system. Nature, 2005, 436: 568-572

55 Ashley C E, Carnes E C, Phillips G K, et al. The targeted delivery of multicomponent cargos to cancer cells by nanoporous particlesupported lipid bilayers. Nat Mater, 2011, 10: 389-397

56 Irvine D J. Drug delivery: One nanoparticle, one kill. Nat Mater, 2011, 10: 342-343

57 Doshi N, Zahr A S, Bhaskar S, et al. Red blood cell-mimicking synthetic biomaterial particles. Proc Natl Acad Sci USA, 2009, 106: 21495-21499

58 Merkel T J, Jones S W, Herlihy K P, et al. Using mechanobiological mimicry of red blood cells to extend circulation times of hydrogel microparticles. Proc Natl Acad Sci USA, 2011, 108: 586-591

$59 \mathrm{Hu} \mathrm{C} \mathrm{M}$, Zhang L, Aryal S, et al. Erythrocyte membranecamouflaged polymeric nanoparticles as a biomimetic delivery platform. Proc Natl Acad Sci USA, 2011, 108: 10980-10985

60 Wong C, Stylianopoulos T, Cui J, et al. Multistage nanoparticle delivery system for deep penetration into tumor tissue. Proc Natl Acad Sci USA, 2011, 108: 2426-2431

61 von Maltzahn G, Park J H, Lin K Y, et al. Nanoparticles that communicate in vivo to amplify tumour targeting. Nat Mater, 2011, 10: $545-552$

62 Tasciotti E, Liu X, Bhavane R, et al. Mesoporous silicon particles as a multistage delivery system for imaging and therapeutic applications. Nat Nanotechnol, 2008, 3: 151-157

63 Sakamoto J, Annapragada A, Decuzzi P, et al. Antibiological barrier nanovector technology for cancer applications. Expert Opin Drug Deliv, 2007, 4: 359-369 
64 Bayliss S C, Heald R, Fletcher D I, et al. The culture of mammalian cells on nanostructured silicon. Adv Mater, 1999, 11: 318-321

65 Chin V, Collins B E, Sailor M J, et al. Compatibility of primary hepatocytes with oxidized nanoporous silicon. Adv Mater, 2001, 13: 1877-1880

66 Canham L. Bioactive silicon structure fabrication through nanoetching techniques. Adv Mater, 1995, 7: 1033-1037

67 Canham L, Reeves C L, Newey J, et al. Derivatized mesoporous silicon with dramatically improved stability in simulated human blood plasma. Adv Mater, 1999, 11: 1505-1507

68 Decuzzi P, Lee S, Decuzzi M, et al. Adhesion of microfabricated particles on vascular endothelium: A parametric analysis. Ann Biomed Eng, 2004, 32: 793-802

69 Decuzzi P, Ferrari M. The adhesive strength of non-spherical particles mediated by specific interactions. Biomaterials, 2006, 27: 5307-5314

70 Gentile F, Curcio A, Indolfi C, et al. The margination propensity of spherical particles for vascular targeting in the microcirculation. J Nanobiotechnol, 2008, 6: 9

71 Gentile F, Chiappini C, Fine D, et al. The effect of shape on the margination dynamics of non-neutrally buoyant particles in twodimensional shear flows. J Biomech, 2008, 41: 2312-2318

72 Gentile F, Ferrari M, Decuzzi P. The transport of nanoparticles in blood vessels: The effect of vessel permeability and blood rheology. Ann Biomed Eng, 2008, 36: 254-261

73 Lee S Y, Ferrari M, Decuzzi P. Shaping nano-/micro-particles for enhanced vascular interaction in laminar flows. Nanotechnology, 2009, 20: 495101

74 Lee S Y, Ferrari M, Decuzzi P. Design of bio-mimetic particles with enhanced vascular interaction. J Biomech, 2009, 42: 1885-1890

75 Ananta J S, Godin B, Sethi R, et al. Geometrical confinement of gadolinium-based contrast agents in nanoporous particles enhances $\mathrm{t} 1$ contrast. Nat Nanotechnol, 2010, 5: 815-821

76 Tanaka T, Mangala L S, Vivas-Mejia P E, et al. Sustained sirna delivery by mesoporous silicon particles for cancer treatment. Cancer Res, 2010, 70: 3687-3696

77 Serda R E, Mack A, Pulikkathara M, et al. Cellular association and assembly of a multistage delivery system. Small, 2010, 6: 1329-
1340

78 Serda R E, Mack A, van de Ven A L, et al. Logic-embedded vectors for intracellular partitioning, endosomal escape, and exocytosis of nanoparticles. Small, 2010, 6: 2691-2700

79 Serda R E, Ferrati S, Godin B, et al. Mitotic trafficking of silicon microparticles. Nanoscale, 2009, 1: 250-259

80 Shen H, You J, Zhang G, et al. Cooperative, nanoparticle-enabled thermal therapy of breast cancer. Adv Healthc Mater, 2012, 1: 84-89

81 Tanaka T, Mangala L S, Vivas-Mejia P E, et al. Sustained small interfering RNA delivery by mesoporous silicon particles. Cancer Res, 2010, 70: 3687-3696

82 Godin B, Gu J, Serda R E, et al. Tailoring the degradation kinetics of mesoporous silicon structures through pegylation. J Biomed Mater Res A, 2010, 94: 1236-1243

83 Tasciotti E, Godin B, Martinez J O, et al. Near-infrared imaging method for the in vivo assessment of the biodistribution of nanoporous silicon particles. Mol Imaging, 2011, 10: 56-68

84 Tanaka T, Godin B, Bhavane R, et al. In vivo evaluation of safety of nanoporous silicon carriers following single and multiple dose intravenous administrations in mice. Int J Pharm, 2010, 402: 190-197

85 Decuzzi P, Godin B, Tanaka T, et al. Size and shape effects in the biodistribution of intravascularly injected particles. J Control Release, 2010, 141: 320-327

86 van de Ven A L, Kim P, Haley O, et al. Rapid tumoritropic accumulation of systemically injected plateloid particles and their biodistribution. J Control Release, 2011, doi: http://dx.doi.org/10. 1016/j.jconrel.2011.10.021

87 Mann A P, Tanaka T, Somasunderam A, et al. E-selectin-targeted porous silicon particle for nanoparticle delivery to the bone marrow. Adv Mater, 2011, 23: H278-H282

88 De Rosa E, Chiappini C, Fan D, et al. Agarose surface coating influences intracellular accumulation and enhances payload stability of a nano-delivery system. Pharm Res, 2011, 28: 1520-1530

89 Fan D, De Rosa E, Murphy M B, et al. Mesoporous silicon-PLGA composite microspheres for the double controlled release of biomolecules for orthopedic tissue engineering. Adv Funct Mater, 2012, 22: 282-293

Open Access This article is distributed under the terms of the Creative Commons Attribution License which permits any use, distribution, and reproduction in any medium, provided the original author(s) and source are credited. 